\title{
Praxismessung versus Selbstmessung: Wie sich die Blutdruckwerte unterscheiden
}

\author{
Für die häusliche Blutdruckmessung \\ gibt es bislang keine zuverlässigen \\ Grenzwerte, die eine Vergleichbar- \\ keit mit den Blutdruckmessungen in \\ der Praxis herstellen. Die mangelnde \\ prognostische Aussagekraft war \\ möglicherweise einer der Gründe \\ für die bislang noch immer einge- \\ schränkte Bedeutung der Selbstmes- \\ sung.
}

— In der vorliegenden Arbeit wurden Blutdruckbereiche für die Selbstmessung definiert, die von ihrem kardiovaskulären Risiko her den für die Praxisblutdruckmessung definierten Stadien entsprachen: Prähypertonie Stadium I (120/80 mmHg), Prähypertonie Stadium II (130/85 mmHg), Hypertonie Stadium I (140/90 mmHg) und Hypertonie Stadium II (160/100 mmHg). Die Messung fand an 6470 Probanden statt (mitleres Alter: 59,3 Jahre; 56,9\% Frauen; $22,4 \%$ unter antihypertensiver Therapie). Es wurden jeweils Grenzwerte für die häusliche Blutdruckmessung bestimmt, die vom kardiovakulären 10-Jahres-Risiko her dem der oben aufgeführten Hypertoniestadien der Praxisblutdruckmessung entsprachen. Die mediane Verlaufsbeobachtung betrug 8,3 Jahre.

Die risikoadaptierten Stadien für die Selbstmessung wurden jeweils am Gesamtkollektiv und für die unbehandel-

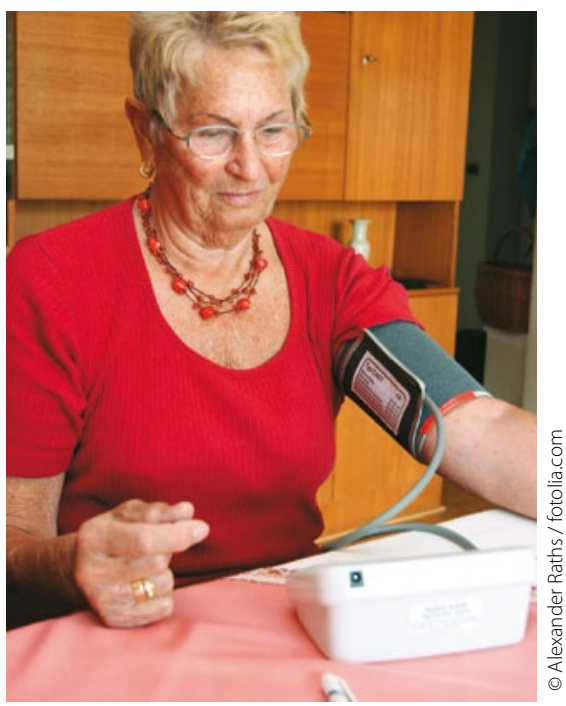

Was zu Hause gut aussieht, kann in der Praxis eine handfeste Hypertonie sein.

ten Teilnehmer ermittelt. Ferner wurden für die Ermittlungen der risikoadaptierten Stadien sowohl das gesamte kardiovaskuläre Risiko als auch das Schlaganfallrisiko und das kardiale Risiko zugrunde gelegt.

Selbst gemessene 119/77 mmHg entsprachen demnach einer Praxismessung von $120 / 80 \mathrm{mmHg}, 125 / 80 \mathrm{mmHg}$ waren äquivalent mit 130/85 $\mathrm{mmHg}$ in der Praxis, 132/82 $\mathrm{mmHg}$ waren gleich $140 / 90 \mathrm{mmHg}$ und $145 / 88 \mathrm{mmHg}$ entsprachen einer Hypertonie Stadium II (160/100 mmHg in der Praxismessung). Ähnliche Blutdruckbereiche wurden auch für das Schlaganfallrisiko und das kardiale Risiko ermittelt.

\section{Kommentar}

Für die Praxis ist bedeutsam, dass die Grenzwerte für die Blutdruckselbstmessung offenbar niedriger liegen als bisher angenommen. Die gegenwärtigen Leitlinien gehen davon aus, dass dem Praxisblutdruck von 140/90 mmHg bei der Selbstmessung 135/85 mmHg entsprechen. In der vorliegenden Arbeit kommen die Autoren aber zu einem deutlich niedrigeren Wert, nämlich etwa 132/82 $\mathrm{mmHg}$, der dem Praxisblutdruck von 140/90 $\mathrm{mmHg}$ entspricht.

Die vorliegende Arbeit ist ein wichtiger Schritt, um verlässliche Grenzwerte für die Blutdruckselbstmessung zu entwickeln. Die meisten Studien in der Vergangenheit hatten ergeben, dass der prognostische Wert der Selbstmessung hinsichtlich kardiovaskulärer Ereignisse deutlich über dem der Praxismessung liegt. Deswegen ist diese Arbeit ein wichtiger Schritt hin zu einer verlässlichen Vergleichsgrundlage.

W. ZIDEK

- T. J. Niiranen et al.

Outcome-Driven Thresholds for Home Blood Pressure Measurement. International Database of Home blood pressure in relation to Cardiovascular Outcome. Hypertension 2012; doi:10.1161/hypertensionaha.111.00100 